\title{
Expression and Distribution Pattern of Retinoic Acid Receptors in the Nasal Mucosa
}

\author{
Jiwon Kwak, MD, Tae Hoon Lee, MD, Munsoo Han, MD, \\ Sang Hag Lee, MD, PhD, and Tae Hoon Kim, MD, PhD \\ Department of Otorhinolaryngology-Head \& Neck Surgery, College of Medicine, Korea University, Seoul, Republic of Korea
}

Background and Objectives: Retinoids are naturally occurring vitamin A derivatives that regulate cellular processes and metabolism. In particular, retinoids play a key role in cellular proliferation by binding to retinoic acid receptors (RAR)-alpha, beta, and gamma. Considering the functional role of nasal mucosa where active cell regeneration occurs, RAR may play a role in tissue remodeling of the human nasal mucosa.

Methods: In this study, we investigated the expression and distribution pattern of RAR using reverse transcription-polymerase chain reaction (RT-PCR), immunohistochemistry (IHC) and Western blot in normal ethmoid mucosa (NE), chronic rhinosinusitis (IE) and polyp (P).

Results: IE and P samples showed higher expression levels of RAR in RT-PCR and Western blot than NE samples. RAR reactivity was also observed in the NE group, which indicates that cell regeneration also occurs in normal condition. Through IHC, we found the localization of RAR. RAR- $\alpha$ was distributed in the epithelial cells, submucosal glands, and endothelial cells. RAR- $\beta$ was located in the basal epithelium, while RAR- $\gamma$ was present in goblet cells and submucosal glands. The staining intensity of RAR- $\alpha, \beta$ and $\gamma$ was higher than that in the NE group. Especially in the P group, RARs were abundantly distributed in the stalks of polyps.

Conclusion: The stalk region contains a lot of collagen and fibroblasts to support polyp formation, and the greater amount of RAR in the stalk suggested that RARs may be associated with angiogenesis and cell proliferation. Accordingly, elevated RAR levels in chronic rhinosinusitis could indicate that RARs play a critical role in cell regeneration, angiogenesis and immunomodulation under inflammatory conditions in the human nasal mucosa.

Keywords: Retinoic acid receptor; Sinusitis; Nasal polyp; Retinoic acid.

\section{INTRODUCTION}

Retinoids are naturally occurring vitamin A derivatives that regulate cellular processes and metabolism [1]. In cell differentiation, specific retinoids are involved in the control of the cell cycle, cell growth and response to cell impairment [2]. More specifically, retinoic acids are involved in epithelial proliferation and differentiation, angiogenesis, and mucus secretion $[3,4]$. Particularly, retinoids have essential, pleiotropic effects on cellular proliferation and differentiation, and they

Received: August 19, 2021 Revised: September 27, 2021

Accepted: October 1, 2021

Address for correspondence: Tae Hoon Kim, MD, PhD, Department of Otorhinolaryngology-Head \& Neck Surgery, College of Medicine, Korea University, 73 Goryeodae-ro, Seongbuk-gu, Seoul 02841, Republic of Korea

Tel: +82-2-920-5486, Fax: +82-2-925-5233, E-mail: doctorthk@gmail.com This is an Open Access article distributed under the terms of the Creative Commons Attribution Non-Commercial License (https://creativecommons.org/licenses/by$\mathrm{nc} / 4.0$ ) which permits unrestricted non-commercial use, distribution, and reproduction in any medium, provided the original work is properly cited. exert their cellular effects by binding to the retinoic acid receptors (RAR) $-\alpha,-\beta$, and $-\gamma[5]$.

In various normal human mucosa surfaces, each retinoid receptor is distributed in different sites of organs. RAR- $\alpha$ is commonly located in the epithelial cells of buccal, gingival, palate, and prostate mucosae [6]. RAR- $\beta$ is usually found in the basal epithelial layer of prostate mucosa, while RAR- $\gamma$ lines the granular layer of the gingival epithelium [7]. A common characteristic of these sites is their active regeneration under normal conditions.

The human nasal mucosa is the first barrier against foreign substances and a conditioner for the inhaled air. The nasal mucosa is separated by a basement membrane into epithelium and lamina propria. A pseudostratified columnar epithelium is composed of four cell types, including goblet cells and basal cells, which are involved in the preservation of the nasal mucosa $[8,9]$. In numerous nasal airway diseases, such as rhinitis and acute and chronic sinusitis, the pseudostratified epi- 
thelium protects against damage, and it regenerates to restore its previous function [9]. In the chronic inflammatory condition of the nasal cavity, the dynamic process of tissue remodelling results in histological changes in the paranasal sinus mucosa. This process includes fibroblast proliferation, angiogenesis, and degradation and formation of subepithelial tissues, which result in fibrosis [10].

Here, we hypothesized that RARs play a role in mucosal regeneration and tissue remodelling of the human nasal mucosa, and accordingly, investigated their expression and distribution pattern in normal ethmoid sinus mucosa (NE), inflammatory ethmoid sinus mucosa (IE), and nasal polyp (P) using reverse transcription-polymerase chain reaction (RT-PCR), immunohistochemistry (IHC), and western blot analysis.

\section{METHODS}

\section{Sample preparation}

Samples of NE $(n=15)$ were obtained from the ethmoid sinuses of patients with blowout fracture during endoscopic reduction surgery. Participants with no history of nasal infections, allergic rhinitis, and previous medical treatment were selected. Patients with a smoking history were excluded, as it may have an intranasal effect. For the control group, normalappearing sinus mucosa with no traumatic injury was extracted. IE samples $(n=15)$ were obtained from 15 patients who underwent endoscopic sinus surgery for chronic sinusitis with P. A history of asthma, nasal allergy, and aspirin sensitivity was an exclusion criterion for the experimental group. None of these patients had ongoing drug treatment. Before sample extraction, all patients signed an informed consent form approved by the Institutional Review Boards of Korea University Anam Hospital (2018AN0061).

Tissue samples (15 NE and 15 IE) were cut into two parts; one part was used for RNA isolation, while the other was used for protein extraction. The tissue samples for RNA isolation were stored at $-80^{\circ} \mathrm{C}$ in liquid nitrogen until RNA extraction. The tissue samples for protein extraction were fixed overnight with $4 \%$ paraformaldehyde in phosphate-buffered saline (PBS, $\mathrm{pH}$ 7.4) for IHC. Then, these were dehydrated in a graded series of ethanol to xylene and embedded in paraffin wax.

\section{Total RNA isolation, RT-PCR, and semiquantitative RT-PCR}

After total RNA $(1 \mu \mathrm{g})$ from each sample was isolated, reverse transcription was done in $20 \mu \mathrm{L}$ of a reaction mixture containing 2.5 U of Molony murine leukemia virus reverse transcriptase (RT; Gibco BRL, Grand Island, NY, USA). The $50 \mathrm{pmol}$ of random hexanucleotides were at $42^{\circ} \mathrm{C}$ for $60 \mathrm{~min}$. The amplified transcripts of glyceraldehyde-3-phosphate de-
Table 1. Sequences of primers used for semiquantitative reverse transcription-polymerase chain reaction

\begin{tabular}{ll}
\hline RAR- $\alpha$ & S: 5'-ATGGCCAGCAACAGCAGCTCCTGCCCGA-3' \\
& AS: 5'-CAGCCCCGTCTCCGCATCAT-3' \\
RAR- $\beta$ & S: 5'-TCGGGGCTGGGAAAAAGACC-3' \\
& AS: 5'-GCTAGGACTGTGCTCTGCTG-3' \\
RAR- $\gamma$ & S: 5'-CAGGGGACTCTCACACCGCA-3' \\
& AS: 5'-GCAGATGAGGCAGATGGCGC-3' \\
GAPDH & S: 5'-ATCTTCCAGGAGCGAGATCC-3' \\
& AS: 5'-ACCACTGACACGTTGGCAGT-3' \\
\hline
\end{tabular}

RAR, retinoic acid receptors; GAPDH, glyceraldehyde-3-phosphate dehydrogenase

hydrogenase (GAPDH) were used as an internal control to determine the quality and quantity of RNA. A negative control was set up by omitting the RT enzyme from the complementary DNA (cDNA) synthesis for each specimen. Table 1 shows the primer sequences used in this analysis. In $2 \%$ agarose gels stained with ethidium bromide, each amplified PCR products were resolved, and photographed with ultraviolet light. The identity of PCR products was confirmed by comparing their corresponding mRNA sequences with the gene sequences deposited in the National Center for Biotechnology Information database.

Thereafter, a semiquantitative PCR was performed to assess the relative expression of RAR- $\alpha,-\beta$, and $-\gamma$ mRNA in NE, IE, and P. The optimal number of PCR cycles for each cDNA samples was determined by plotting the PCR product yield of different cycles on a semi-logarithmic graph; the cycle number that represented the exponential amplification was selected for final amplification. The PCR products were quantified by analysing the bands of the samples via densitometry. The ratio of cDNA and its corresponding GAPDH cDNA was calculated. Data are presented as mean \pm standard deviation (SD).

To evaluate the statistical significance of differences between the groups, Mann-Whitney U test was used, and the level of statistical significance was set at $\mathrm{p}<0.05$.

\section{Immunohistochemical and western blot analyses}

Immunohistochemical staining was performed with a peroxidase-labelled streptavidin-biotin assay to evaluate the expression and distribution of RAR- $\alpha,-\beta$, and $-\gamma$ in NE, IE, and P. Briefly, tissue sections were deparaffinized, rehydrated, using 3\% hydrogen peroxidase in methanol for $15 \mathrm{~min}$ to quench the endogenous peroxidase activity. After washing in $10 \mathrm{mM}$ PBS ( $\mathrm{pH}$ 7.4), the sections were incubated with 1:100 dilutions of rabbit anti-RAR- $\alpha,-\beta$, and $-\gamma$ polyclonal antibodies (Santa Cruz Biotechnology, CA, USA) overnight at room temperature. The color was developed using 3, 3'-diaminobenzidine.

For western blotting, the total protein was separated on $12 \%$ 
sodium dodecyl sulphate-polyacrylamide gels and transferred onto immobilon (Millipore, Bedford, MA, USA). Then with Tris-buffered saline containing $1 \%$ skim milk, the blots were blocked, for $1 \mathrm{~h}$, then incubated with appropriate antibodies in Tris-buffered saline overnight. This process was done at room temperature. Using an ECL detection kit (Amersham Bioscience, Piscataway, NJ, USA), antibody reactions were detected, followed by the detection of chemiluminescence with an $\mathrm{x}$-ray film. As an internal control, analysis of $\beta$-actin expression was performed in parallel blots using $\beta$-actin antibodies (Sigma, St. Louis, MO, USA). To quantify the intensity of the detected bands, the scion image Beta 4.0.2 (Scion Corporation, Frederick, MD, USA) was used. The relative signal intensity of each protein was obtained by dividing the intensity of each protein by the signal of $\beta$-actin. The statistical significance of their differences was evaluated using Mann-Whitney $U$ test, and the level of statistical significance was set at $\mathrm{p}<0.05$.

\section{RESULTS}

\section{RAR mRNA expression analysis in NE, IE, and P samples via RT-PCR}

Using semiquantitative RT-PCR analysis, the mRNA expression of RAR- $\alpha,-\beta$, and $-\gamma$ and GAPDH was detected in the clinical NE, IE, and P samples. As a positive control, all samples were analysed for GAPDH and showed an expected size of 502 bp. Fig. 1 shows the result of the semiquantitative RT-PCR after normalization with $\beta$-actin expression, reveal- ing the expression of RAR- $\alpha,-\beta$, and $-\gamma$ mRNAs. As shown in Fig. $1 \mathrm{~A}, \mathrm{RAR}-\alpha,-\beta$, and $-\gamma$ were all expressed in NE, IE, and P; the expression of RAR- $\alpha,-\beta$, and $-\gamma$ mRNAs was detected in all samples examined corresponding to each expected size. Nevertheless, the relative abundance of RAR- $\alpha,-\beta$, and $-\gamma$ was significantly higher in the IE and $\mathrm{P}$ group than in the NE group (Fig. 1B).

\section{Immunohistochemical localization and western blotting of RARs in NE, IE, and P samples}

The tissues were subjected to IHC staining using specific antibodies against RAR- $\alpha,-\beta$, and $-\gamma$. In the NE group, RAR- $\alpha$ was localized in epithelial cells, submucosal glands, and endothelial cells (Fig. 2A). In the IE and P groups, the detected localization site was the same as that in the NE group, while the staining intensity was higher than that in the NE group. Specifically, in the P group, the staining of endothelial cells was more positively distributed in the stalk area, which has more vessels, than in the body. RAR- $\beta$ immunoreactivity was detected on the basal layer of the epithelium in the NE group, while more intense staining was detected in the IE and $P$ groups (Fig. 2B). In the IE and P groups, RAR- $\beta$ was also distributed in the endothelial cells. The expression pattern of RAR- $\gamma$ was the same in all samples, and it was detected in goblet cells and submucosal glands. Similar to other receptors, its immunoreactivity was more intense in the IE and P groups (Fig. 2C). The $P$ samples showed abundant RAR- $\alpha$ and $-\beta$ staining in the endothelial cells, particularly in the stalk area.

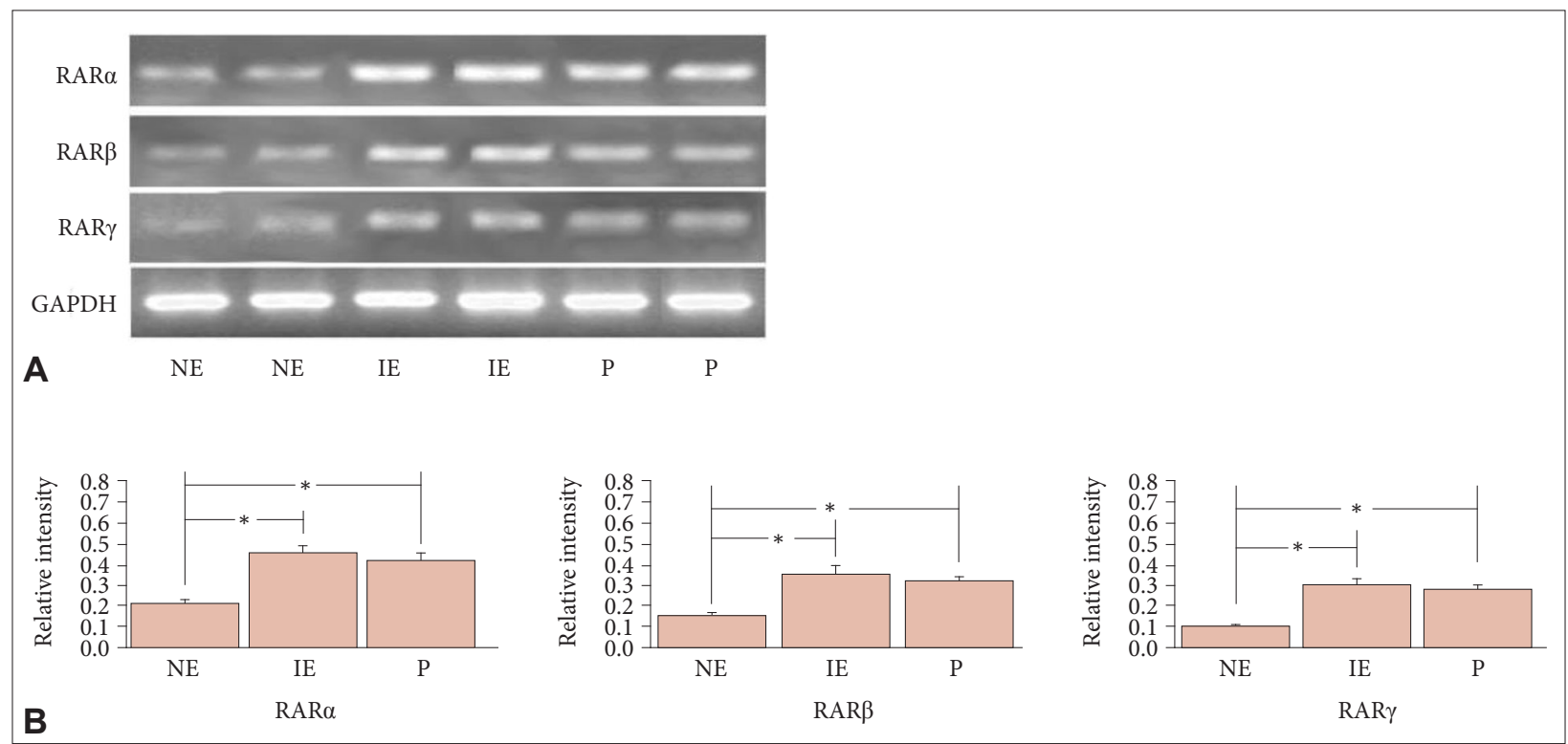

Fig. 1. Semiquantitative RT-PCR of NE, IE and P samples. A: Semiquantitative RT-PCR analysis of RAR mRNA levels in normal nasal mucosa and nasal mucosa with chronic sinusitis and polyps. B: Bands were quantified via densitometric scanning, and the expression of each gene was calculated as relative to that of the internal control GAPDH. The bar indicates mean \pm standard deviation. * $\mathrm{p}<0.05$. RT-PCR, reverse transcription-polymerase chain reaction; RAR, retinoic acid receptors; GAPDH, glyceraldehyde-3-phosphate dehydrogenase; NE, normal ethmoid sinus mucosa; IE, inflammatory ethmoid sinus mucosa; P, nasal polyp. 

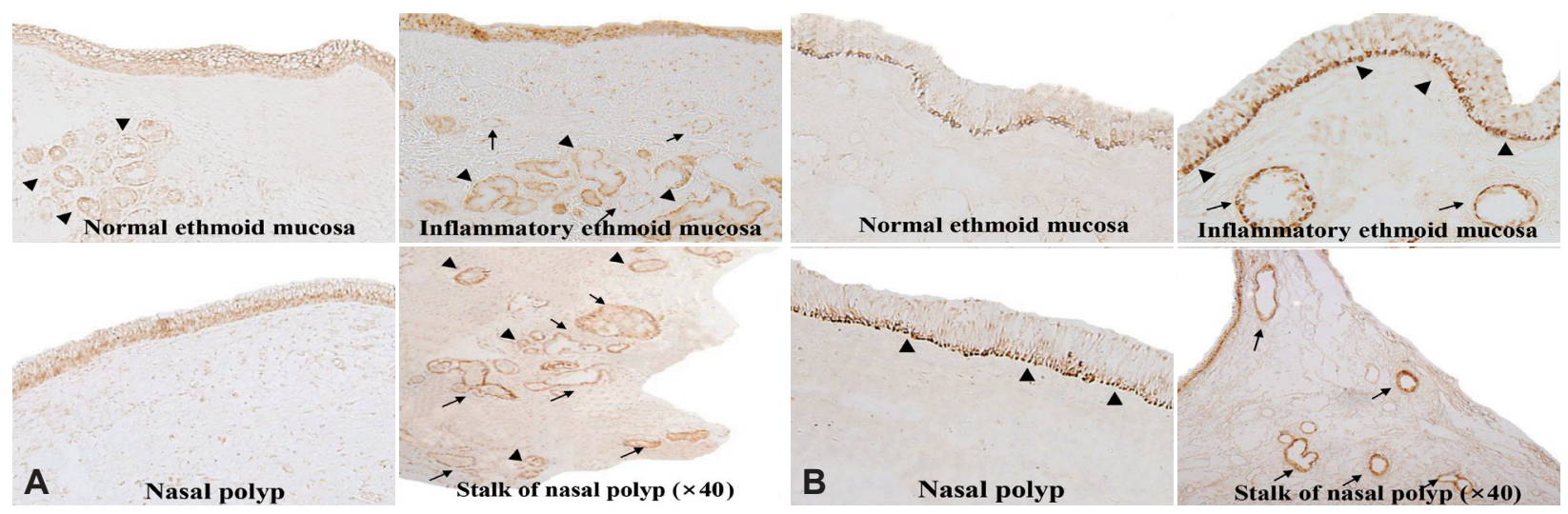

A

Nasal polyp
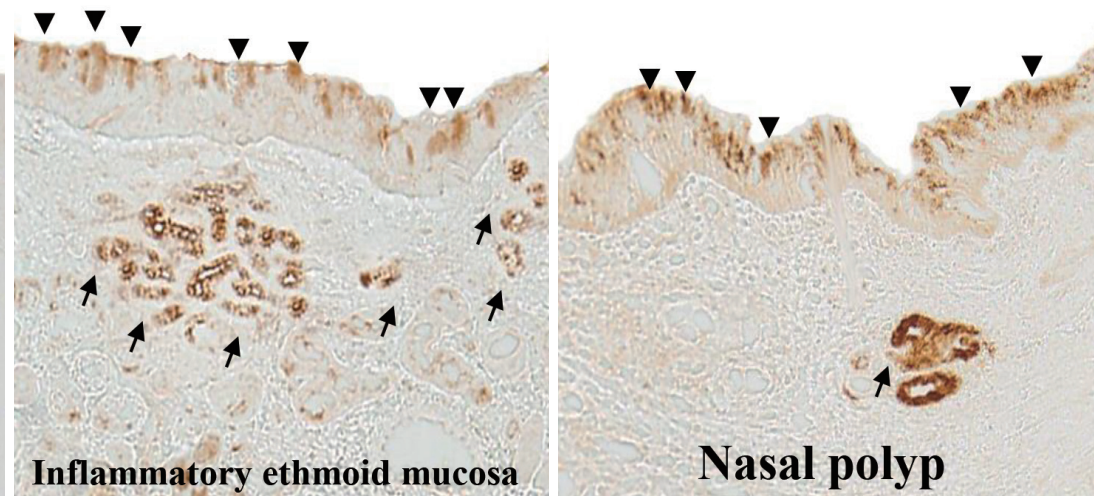

\section{Normal ethmoid mucosa}

Fig. 2. Immunohistochemical localization of RAR- $\alpha$ in normal mucosa, inflammatory ethmoid mucosa, and nasal polyp and its stalk. A: RAR expression was observed in epithelial cells (arrowheads) and submucosal glands (arrows) $(\times 100)$. B: Expression pattern of RAR- $\beta$ based on immunohistochemical analysis as shown in (A) $(\times 100)$ (epithelial cells [arrowheads] and endothelial cells [arrows]). C: RAR- $\gamma$ localization based on immunohistochemical analysis (×400). RAR expression in epithelial cells (arrowheads) and submucosal glands (arrows). RAR, retinoic acid receptors.

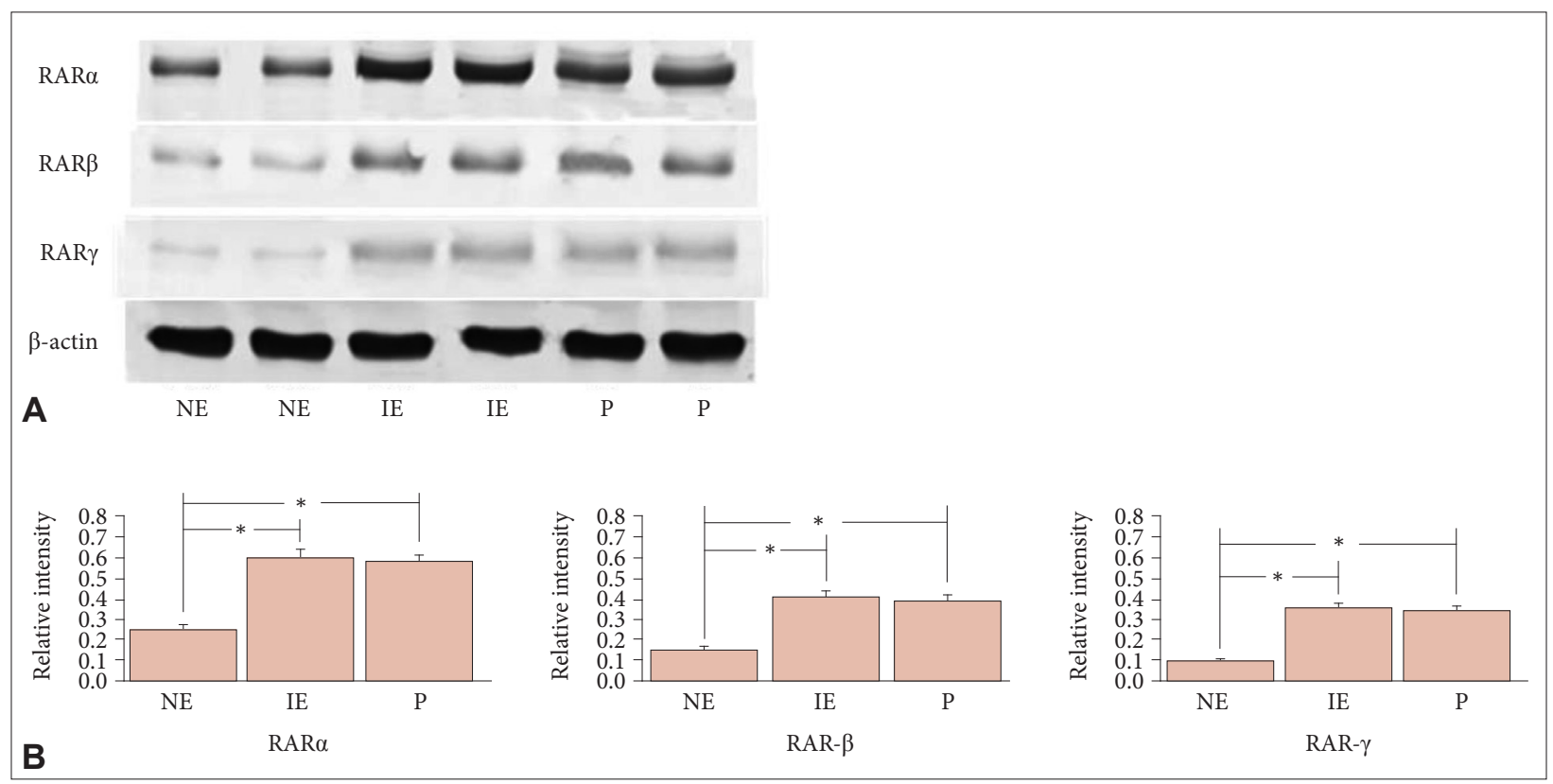

Fig. 3. Western blot of NE, IE and P samples. A: Western blot analysis of RAR- $\alpha,-\beta$, and $-\gamma$ in normal mucosa, inflammatory nasal mucosa, and nasal polyp. B: Bands were quantified via densitometric scanning, and the expression of each gene was calculated as relative to that of the internal control $\beta$-actin. The bar indicates mean \pm standard deviation. ${ }^{*} p<0.05$. RAR, retinoic acid receptors; NE, normal ethmoid sinus mucosa; IE, inflammatory ethmoid sinus mucosa; P, nasal polyp. 
Next, we performed western blotting to analyse RAR- $\alpha,-\beta$, and $-\gamma$ protein expression in the NE, IE, and P samples. The expression levels of RAR- $\alpha$, $-\beta$, and $-\gamma$ in all samples were significantly higher in the IE and P samples than in the NE sample (Fig. 3) $(\mathrm{p}<0.05)$.

\section{DISCUSSION}

To gain an understanding of the role of RARs on NE, IE, and $\mathrm{P}$, the expression and distribution pattern of RARs were determined. RT-PCR and western blot analysis were used to determine the expression levels of RARs in each group, and IHC was performed to investigate their distribution pattern. The RARs located in the nasal mucosa were more intensely expressed in chronic rhinosinusitis, which is an inflammatory condition of the nasal cavity. This finding was verified at both mRNA and protein levels using RT-PCR and western blotting, respectively. The results of immunohistochemical staining revealed that RAR- $\alpha$ was distributed in the epithelial cells, submucosal glands, and endothelial cells. RAR- $\beta$ was located in the basal epithelium, while RAR- $\gamma$ was present in the goblet cells and submucosal glands. All immunohistochemical signals of RAR- $\alpha,-\beta$, and $-\gamma$ were stronger in the IE and P samples than in the NE sample. Taken together, these data suggest that RARs are constitutively expressed in NE and are intensely immunoreactive in IE. There are few studies about the relationship between each RAR subtype and the nasal mucosa. Although it is well-known that active cell renewal and division occur in the nasal mucosa, this study is the first to explore the role of RARs in the nasal mucosa.

As the nasal mucosa is the first barrier in the respiratory tract, the sinus mucosa is subjected to frequent cell damage and is characterized by newly regenerated tissues, which are peeled off in inflammatory conditions [11]. According to a study in a rabbit model, topical retinoic acid gels help in the regeneration of the sinus mucosa, which suggests that topical retinoic acids can promote sinus wound healing [12]. A normal sinus mucosa is considered to be an active site of cell cycling, and retinoic acid activity can be detected in normal conditions. Consistently, the expression of RARs confirmed via RT-PCR and western blotting was also observed in NE, where cell regeneration occurs.

In general, retinoic acid is metabolized in the small intestine and exists in high concentrations in organs such as the eye. At high levels, it plays a key role in mucosal immunity and immune tolerance, while at basal levels, it is involved in cell survival and immune cell activation [13]. Retinoic acid can also suppress the growth and angiogenesis of tumours and prevent their metastasis [14]. Based on its biological mechanism, some studies found that retinoic acid can be used for the treatment of several diseases. Indeed, the U.S. Food and Drug Administration approved retinoic acid as a treatment for lymphoma and leukaemia. Other studies also stated that retinoic acid is effective for the treatment of malignant diseases, such as prostate cancer, breast cancer, melanoma, and hepatoma [7-14]. These findings suggest that retinoic acid controls cell proliferation and prevents tumour progression, which is in contrast to our hypothesis that retinoic acid promotes cell generation and proliferation. Other studies focused on the up-regulated activity of retinoic acid with its ligand in inflammatory conditions, such as in the synovial membrane with osteoarthritis (OA) or rheumatoid arthritis (RA) [15]. In one study, RAR expression was found to be increased in the synovial membranes of patients with OA or RA. The RARs expressed in an inflammatory condition evoked cartilage destruction and up-regulate inflammatory cytokines and matrix metalloproteinases. Accordingly, the elevated RAR levels in chronic rhinosinusitis could indicate that RARs play a critical role in immunomodulation under inflammatory conditions.

In $\mathrm{P}$, angiogenesis is an important process in polyp formation [16]. It has been demonstrated that angiogenic chemokines such as vascular endothelial growth factor (VEGF), angiopoietin 1 and -2 (Ang-1, Ang-2), and stromal cell-derived factor- $1 \alpha, \beta$ (SDF-1 $\alpha$, SDF-1 $\beta$ ) are more likely to be expressed in polyposis mucosa [17]. Likewise, anti-angiogenic agents, such as bevacizumab that inhibit VEGF, are considered to be a future treatment for polyps. VEGF is important for angiogenesis in the polyp stalk; hence, it is a critical area in polyp formation [18]. Moreover, the polyp stalk has a greater amount of collagen and fibroblasts, which supports its role in polyp formation. This study shows that RARs are abundantly distributed in the stalk region of polyps, suggesting that elevated levels of RARs may be associated with angiogenesis and cell proliferation.

Nevertheless, this study has some limitations. Since the results only showed the expression levels of the RARs and not retinoic acid itself, it cannot be concluded that retinoic acid is also activated in the nasal mucosa. Further studies are needed to confirm the direct activity of retinoic acid in a NE and in the mucosa of a patient with chronic sinusitis. Moreover, this study only showed the pattern of RAR expression and distribution, but the causal mechanisms were not revealed. As mentioned above, RAR may be involved in cell regeneration or aberrant cell proliferation regarding cancer cell metastasis. Furthermore, it also can be associated with epithelial-mesenchymal transition (EMT) which plays a role in inflammation and tissue remodeling [19]. To elaborate, additional studies such as wound healing assays should be conducted in the future to confirm that whether RARs play a role in cell proliferation. Studies about biomarkers of EMT like vimentin, E-cad- 
herin and N-cadherin could be helpful to clarify the association between RAR and EMT. Also the aberrant RAR phosphorylation itself could induce the deregulated expression of RAR target gene resulting in elevation of cancer risk. Thus such molecular-level further studies will help us understand the role of RAR in the nasal cavity.

\section{Availability of Data and Material}

All data generated or analyzed during the study are included in this published article.

\section{Conflicts of Interest \\ The authors have no potential conflicts of interest to disclose.}

\section{Author Contributions}

Conceptualization: Tae Hoon Kim. Data curation: Jiwon Kwak, Tae Hoon Lee. Formal analysis: Tae Hoon Kim. Funding acquisition: Tae Hoon Kim. Investigation: Jiwon Kwak. Methodology: Tae Hoon Kim. Project administration: Tae Hoon Kim. Resources: Jiwon Kwak. Software: Jiwon Kwak. Supervision: Tae Hoon Lee, Munsoo Han, Sang Hag Lee. Validation: Jiwon Kwak. Visualization: Jiwon Kwak. Writing_original draft: Jiwon Kwak. Writing_-review \& editing: Jiwon Kwak, Tae Hoon Kim.

\section{ORCID iDs \\ Jiwon Kwak https://orcid.org/0000-0002-1907-0178 \\ Tae Hoon Lee $\quad$ https://orcid.org/0000-0002-7362-6798 \\ Munsoo Han https://orcid.org/0000-0003-1359-6248 \\ Sang Hag Lee https://orcid.org/0000-0001-7698-2707 \\ Tae Hoon Kim https://orcid.org/0000-0001-8811-654X}

\section{Funding Statement}

This research was supported by the Basic Science Research Program, National Research Foundation of Korea, funded by the Ministry of Science and Technology and the Ministry of Science, ICT \& Future Planning (2017 R1A2B2003575, NRF-2020R1A2C1006398), the Ministry of Science and ICT (2020R1C1C1012288), Korea, under the ICT Creative Consilience program (IITP-2021-001819) supervised by the IITP (Institute for Information \& Communications Technology Planning \& Evaluation), the Korea Health Technology R\&D Project (HI17C0387), Korea Health Industry Development Institute (KHIDI), and the Ministry of Health \& Welfare. This research was also supported by a Korea University grant and a grant from Korea University Medical Center and Korea University Anam Hospital, Seoul, Republic of Korea.

\section{REFERENCES}

1. Cassani B, Villablanca EJ, De Calisto J, Wang S, Mora JR. Vitamin A and immune regulation: role of retinoic acid in gut-associated dendritic cell education, immune protection and tolerance. Mol Aspects Med 2012;33(1):63-76.

2. Ziouzenkova O, Plutzky J. Retinoid metabolism and nuclear receptor responses: new insights into coordinated regulation of the PPAR-RXR complex. FEBS Lett 2008;582(1):32-8.

3. Saito A, Sugawara A, Uruno A, Kudo M, Kagechika H, Sato Y, et al. Alltrans retinoic acid induces in vitro angiogenesis via retinoic acid receptor: possible involvement of paracrine effects of endogenous vascular endothelial growth factor signaling. Endocrinology 2007; 148(3):1412-23.

4. Rogers DF, Barnes PJ. Treatment of airway mucus hypersecretion. Ann Med 2006;38(2):116-25.

5. Duong V, Rochette-Egly C. The molecular physiology of nuclear retinoic acid receptors. From health to disease. Biochim Biophys Acta 2011; 1812(8):1023-31.

6. Schön M, Rheinwald JG. A limited role for retinoic acid and retinoic acid receptors RAR alpha and RAR beta in regulating keratin 19 expression and keratinization in oral and epidermal keratinocytes. J Invest Dermatol 1996;107(3):428-38.

7. Campbell MJ, Park S, Uskokovic MR, Dawson MI, Koeffler HP. Expression of retinoic acid receptor-beta sensitizes prostate cancer cells to growth inhibition mediated by combinations of retinoids and a 19-nor hexafluoride vitamin D3 analog. Endocrinology 1998;139(4):1972-80.

8. Scherzad A, Hagen R, Hackenberg S. Current understanding of nasal epithelial cell mis-differentiation. J Inflamm Res 2019;12:309-17.

9. Watelet JB, Van Zele T, Gjomarkaj M, Canonica GW, Dahlen SE, Fokkens $\mathrm{W}$, et al. Tissue remodelling in upper airways: where is the link with lower airway remodelling? Allergy 2006;61(11):1249-58.

10. Radajewski K, Wierzchowska M, Grzanka D, Antosik P, Zdrenka M, Burduk P. Tissue remodelling in chronic rhinosinusitis - review of literature. Otolaryngol Pol 2019;73(5):1-4.

11. Fukazawa K, Ogasawara H, Umemoto M, Sasaki R, Sakagami M. Regeneration of epithelial cells of the maxillary sinus mucosa in chronic sinusitis. Med Electron Microsc 1998;31(1):10-5.

12. Maccabee MS, Trune DR, Hwang PH. Paranasal sinus mucosal regeneration: the effect of topical retinoic acid. Am J Rhinol 2003;17(3):133-7.

13. Kim CH. Chapter four - Retinoic acid, immunity, and inflammation. In: Litwack G, editor. Vitamins and the immune system. 1st ed. Academic Press; 2011. p.83-101.

14. Chen MC, Hsu SL, Lin H, Yang TY. Retinoic acid and cancer treatment. Biomedicine (Taipei) 2014;4(4):22.

15. A Z, Cd K, Ad V, T K, Li S. Expression of retinoic acid receptor (RAR) a protein in the synovial membrane from patients with osteoarthritis and rheumatoid arthritis. Int J Biomed Sci 2007;3(1):46-9.

16. Hirshoren N, Neuman T, Gross M, Eliashar R. Angiogenesis in chronic rhinosinusitis with nasal polyps and in antrochoanal polyps. Inflamm Res 2011;60(4):321-7.

17. Karatzanis AD, Samara KD, Antoniou KM, Lymbouridou R, Chatzakis $\mathrm{N}$, Spandidos DA, et al. Investigation of angiogenetic pathways in nasal polyposis. Mol Med Rep 2012;5(5):1158-62.

18. Nemati S, Keihanian F, Saeidinia A, Bakhshaei M. Effect of bevacizum$\mathrm{ab}$ on growth of human nasal polyposis in vitro; an off-label use of anti-angiogenic agent for nasal polyposis treatment. Drug Des Devel Ther 2019;13:3383-9.

19. Wang M, Sun Y, Li C, Qu J, Zhou B. Eosinophils correlate with epithelial-mesenchymal transition in chronic rhinosinusitis with nasal polyps. ORL J Otorhinolaryngol Relat Spec 2022;84(1):70-80. 